\title{
Administration of serratiopeptidase lead to increase in spread of space infection
}

\author{
Amit Sangle1, Aruna Tambuwala², Gaurav Khutwad ${ }^{3}$, Ashvini Vadane ${ }^{4, *}$ \\ ${ }^{1}$ Guide and Professor, ${ }^{2}$ Professor, ${ }^{3}$ Senior Lecturer, ${ }^{4}$ Post Graduate Student, Dept. of Oral \& Maxillofacial Surgery and \\ Implantology, M.A Rangoonwala Dental College, Pune, Maharashtra, India
}

*Corresponding Author:

Email: drashvinivadane@gmail.com

\begin{abstract}
This article represents a case report in which serratiopeptidase proved a reason to spread of space infection in 32 years old male patient. This case was successfully managed in M.A. Rangoonwala dental college, Pune.
\end{abstract}

Keywords: Serratiopeptidase, Buccal space infection, Submandibular space infection, Submassetric space infection.

\section{Case Report}

A patient $[32$ years/M] visited with chief complaints of pain, swelling and decreased mouth opening. Clinically, we noticed the solitary hard swelling in the lower left back region of jaw, reduced mouth opening $[8 \mathrm{~mm}]$ and carious lower left first molar tooth, suggestive of "left submandibular space infection". We prescribed him cefixime $200 \mathrm{mg}$, metronidazole $400 \mathrm{mg}$, ranitidine $150 \mathrm{mg}$, diclofenac sodium $50 \mathrm{mg}$, paracetamol $500 \mathrm{mg}$.

After 7 days, patient's pain \& swelling was subsided and mouth opening was increased [12 mm]. We extracted offending tooth [36] with minimal trauma, prescribed him previous drugs again for 7 days, except metronidazole $400 \mathrm{mg}$. After this, patient's pain and swelling was subsided.

After 10 days, patient noticed pain, swelling with the same site only and visited some other doctor, who prescribed him diclofenac sodium $50 \mathrm{mg}$, paracetamol $500 \mathrm{mg}$ and serratiopeptidase $15 \mathrm{mg}$ which patient had for unknown period.

After about 45 days, patient visited us with increased swelling, pain with the same site. There was a solitary, hard, fixed swelling of $5 \mathrm{~cm}^{*} 4 \mathrm{~cm}$ in size at the lower left back region of jaw, extending from the rima-oris to the angle of mandible and superiorinferiorly from the line passing from rima-oris to tragus of ear to the inferior border of mandible, "punctum" was also noticed, suggestive of "left submandibular and buccal space infection". Patient's mouth opening was decreased $[11 \mathrm{~mm}]$. Radiographic examination showed features of osteomyelitis. We adviced him to stop previous medications and prescribed cefixime $200 \mathrm{mg}$, metronidazole $400 \mathrm{mg}$, diclofenac sodium $50 \mathrm{mg}$, paracetamol $500 \mathrm{mg}$, ranitidine $150 \mathrm{mg}$ and muscle relaxant containing ibuprofen $400 \mathrm{mg}$, paracetamol 325 $\mathrm{mg}$, chlorzoxazone $250 \mathrm{mg}$.

After 7 days, patient came with pus discharge through the punctum and now the patient was suffering from solitary swelling on the left lateral region of neck below the angle of mandible [hard, fixed, tender, $3 \mathrm{~cm}^{*}$ $2 \mathrm{~cm}$ in size]. This suggested "left submassetric space infection". Radiographically, there was formation of "odontogenic oro-cutaneous fistula" with the extraction socket of 36.

We admitted the patient, administered cefepime $2 \mathrm{gm}+$ Tazobactum $250 \mathrm{mg}$, amikacin sulphate $100 \mathrm{mg}$, ranitidine $50 \mathrm{mg}$, diclofenac $25 \mathrm{mg}$, paracetamol 500 $\mathrm{mg}$, metronidazole $500 \mathrm{mg}$ intravenously. Patient's complete haemogram showed raised "differential leukocyte count". Patient's "Tuberculin test" was performed which was "negative".

On next day, we planned incision and drainage with left submandibular \& buccal space infection. As the swelling present below the angle of mandible, was hard, we did not perform incision and drainage with it. On the next day, this swelling bursted. Then, we explored it. After this, patient's swelling decreased and mouth opening increased, there was absence of pain. After 3 months, radiographic examination revealed complete healing of fistula and patient's mouth opening was increased to $31 \mathrm{~mm}$.

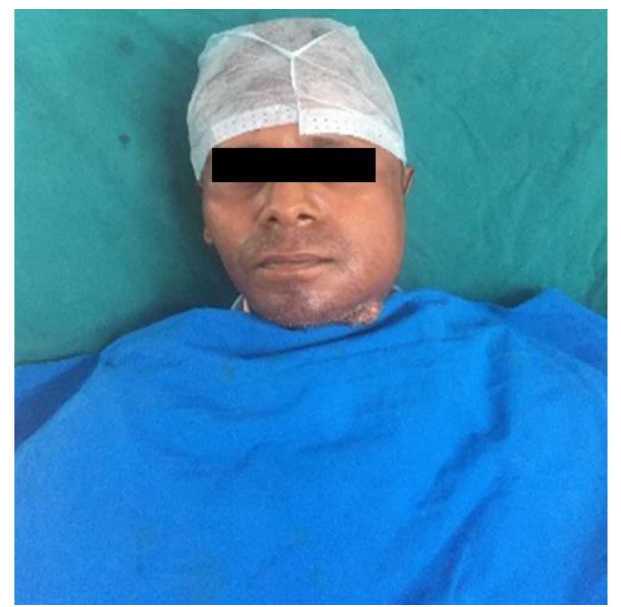

Fig. 1: Pre-operative front profile picture 


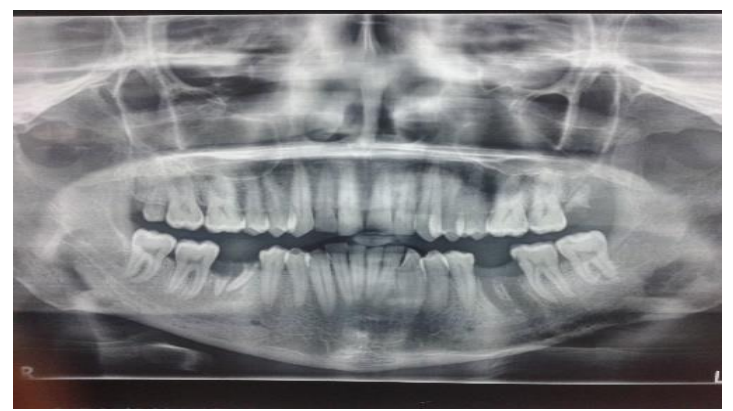

Fig. 2: Pre-operative orthopantamogram [orocutaneous fistula with the extraction socket of 36

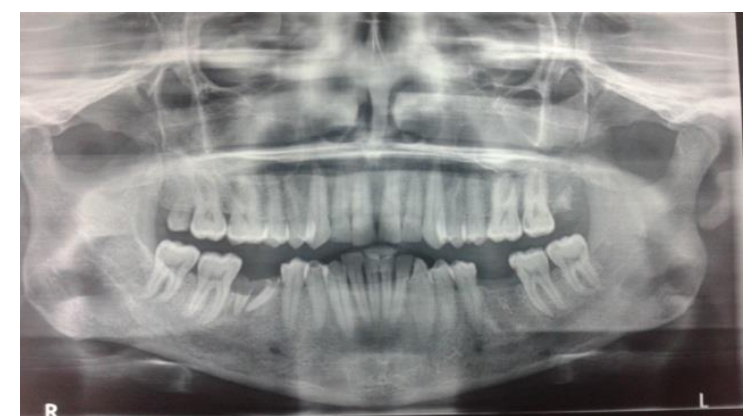

Fig. 3: Orthopantamogram after 3 months [Healing of fistula]

\section{Discussion}

Odontogenic infections are one of the major sources of fascial space infections in the head-neck region, and they are having the potential to spread via fascial spaces. ${ }^{7}$ In this case, the facial spaces affected by the infection are left buccal, submandibular, submassetric spaces.

The involvement of "submasseteric space" by fascial space infection is rare. Very few cases of it are reported. ${ }^{8}$ Surgical incision and drainage under antibiotic coverage is the treatment for fascial space infection. ${ }^{13}$

Osteomyelitis is an inflammatory disease affecting bones. ${ }^{6}$ Osteomyelitis of jaws following dental treatment is rare and mostly it occurs in immunecompromised patients. Hence, we suspected that patient might be suffering from tuberculosis and performed "tuberculin test" but it was "negative".

Radiographic features of osteomyelitis are radiolucency, bony destruction and sequestrum formation. ${ }^{6}$ Orthopantamogram revealed radiolucent areas, bony destruction, formation of fistula from the extraction socket of 36 to the inferior border of mandible. Odontogenic oro-cutaneous fistula is a tract which starts at the apex of an infected tooth or of infected region of the jaw via the alveolar bone and drains all the infection, pus through the skin. ${ }^{9}$

"Serratiopeptidase" is an enzyme which is having proteolytic action. Serratiopeptidase is retrieved from the non-pathogenic enterobacteria serratio E15 which is found in silk-worm. It is used as an anti-inflammatory drug. ${ }^{2}$ Serratiopeptidase causes degradation of insoluble protein products like fibrin and inflammatory mediators. Viscosity of exudates is reduced by the serratiopeptidase, and this facilitates drainage and it relieves pain by inhibiting the bradykinin release. ${ }^{1}$

Any abscess is surrounded by effused $\&$ organized fibrin and inflammatory cells. Serratiopeptidase breaks this fibrin and the pus spreads into the deeper planes. Literature supports that administration of serratiopeptidase causes spread of infection. ${ }^{1}$

\section{Conclusion}

Administration of "serratiopeptidase", in cases of fascial space infections should be limited as it can cause spread of infection.

\section{References}

1. Prashanth Rajaram, Abhishek Bhattacharjee, Smriti Ticku. Serratiopeptidase - A Cause for Spread of Infection. Journal of Clinical \& Diagnostic Research. 2016 Aug, Vol-10 (8) ZD31ZD32.PMCID:PMC5028551.

2. Nirale NM, Menon MD. Topical formulation of serratiopeptidase: Development and pharmacodynamics evaluation. Indian Journal of Pharmaceutical Science. 2010;72(1):65-71.

3. Bhagat S, Agarwal M, Roy V. Serratiopeptidase: A systematic review of the existing evidence:

International Journal of Surgery.2013;11(3):209-17.

4. Johnson BR, Remeikis NA, Yan Cura JE. Diagnosis and treatment of cutaneous facial sinus tracts of dental origin. J Am Dent Assoc. 1999 Jun;130 (6):832 -6.

5. Toshihisa Sato, Hideyunki Suenaga, Musuki Igarashi, Kazuto Hoshi, Tsuyoshi Takasto. Rare case of external dental fistula of the submental region misdiagnosed as inverted follicular keratosis and thyroglossal duct cyst. International Journal of Surgery Case Reports 16 (2015) 39-43.

6. Hemant Mehra, Simit Gupta, Hemant Gupta, V.Sinha, Jasmeet Singh. Chronic Suppurative Osteomyelitis of Mandible: A Case Report. Craniomaxillofac Trauma Reconstruction. 2013;6:197-200.

7. Paolo Boscolo - Rizzo, Maria Cristina, Da Mosto. Submandibular Space Infection: a potentially lethal infection. International Journal of Infectious Diseases (2009),13,327-333.

8. Ashutosh Rai, Ruchi Rajput, Rabindra K Khatua, Mangal Singh. Submassetric abscess: A rare head and neck abscess. Indian Journal of Dental Research,22(1),2011.

9. Kani Bilginaylar. Uncommon Oro-cutaneous Fistula of the Jaw Treated with Platelet-Rich Fibrin. Research Gate. Hindawi. Case Reports in Dentistry. Volume 2017, Article ID 7174217.

10. Nafisa Samir, Abdulaziz Al-Mahrezi, Salim alSudairy. Odontogenic Cutaneous Fistula - Report of two cases. Sultan Qaboos Univ Med J. 2011 Feb;11(1):115-118. 